\title{
On the existence of representative volumes for softening quasi-brittle materials- a failure zone averaging scheme
}

\author{
Vinh Phu Nguyen ${ }^{* 1}$, Oriol Lloberas Valls ${ }^{2}$, Martijn Stroeven ${ }^{3}$, Lambertus Johannes Sluys ${ }^{4}$ \\ Delft University of Technology, Faculty of Civil Engineering and Geosciences, P.O. Box 5048, 2600 GA Delft, The Netherlands
}

\begin{abstract}
The concept of the representative volume element (RVE) for softening materials is revised in this contribution. It is demonstrated by means of numerical simulations that there exists a sample which is statistically representative for quasi-brittle materials with random microstructure like concrete. This finding is an important ingredient for homogenization-based multiscale modelling of softening materials.
\end{abstract}

Key words: representative volume element (RVE), quasi-brittle material, softening, multiscale, homogenization

\section{Introduction}

In the past decade, homogenization-based multiscale modelling (by multiscale we mean multiple length scales, multiple time scales are out of the scope of this manuscript) has been a topic of extensive research, see [1, 2, 3, 4] among others and references therein. A concept of crucial importance in this kind of multiscale modelling is the representative volume element (RVE). There is not a single and exact definition of the RVE for an arbitrary heterogeneous material. That might explain the existence of various definitions of the RVE, see [5] for a recent review. Briefly speaking a microscopic sample is considered to be an RVE when (i) an increase in its size does not lead to considerable differences in the homogenized properties, (ii) the microscopic sample is large enough so that the homogenized properties are independent of the microstructural randomness. The issue of existence of the RVE for materials that exhibit strain localization (e.g., concrete, soil), however, has been addressed by a few researchers [6, 7, ,5]. It has been shown in 7, 5. that for softening materials an RVE cannot be found since the material loses statistical homogeneity upon strain localization. This finding, at first glance, seemed to slow down to a certain extent the development of computational homogenization techniques for modelling softening materials. It however has advanced the development of the socalled coupled volume method [8] in which the unit cell is associated to a domain of finite volume (e.g., an element in a finite element context) rendering the method objective to the size of the unit cells. Along this line, there are the method presented in [9], the VMM-based (variational multiscale method) methods [10, 11], the adaptive heterogeneous multiscale method (HMM) [12] and domain decomposition based methods [13]. However, those methods should not be used for problems when the scales are clearly separated due to the prohibitive computational expense which they would lead to.

\footnotetext{
* Corresponding author

${ }^{1}$ V P.Nguyen@tudelft.nl

20 LloberasValls@tudelft.nl

[3] Stroeven@tudelft.nl

${ }^{4}$ L J.Sluys@tudelft.nl
} 
In the past two years homogenization schemes for material layers [14, 15, 16] have been developed in which a traction-separation law (also known as cohesive law) corresponding to a material layer (e.g., thin fibre-epoxy layer) is obtained based upon finite element computations at the microscale wherein the complex microstructure of the material layers have been explicitly modelled. Very recently, in [17] the authors have proved the existence of an RVE for softening material by deriving a traction-separation law (for a macro crack) instead of a stress-strain relation from the microscopic stresses and strains as usually done in standard computational homogenization schemes. Related works on computational homogenization-based multiscale modelling of cracks have also been reported in [18, 19, 20]. In the micromechanics community, homogenization of materials exhibiting discrete cracking e.g., interface debonding in polycrystalline solids has been addressed intensively, refer to, for instance, [21, 22].

Motivated by the work in [17] the contribution of this paper is the numerical demonstration of the existence of an RVE for a class of softening materials with a random microstructure that exhibits damage in an averaged, smeared fashion. This is in contrast to the material utilized in [17] which shows discrete cracking within a regular microstructure. It is emphasized that the RVE's existence proof is an important step in the process of developing a homogenization scheme for softening materials with a microstructural length scale being very small compared to the macroscopic one. In this fashion, the separation-of-scales principle still applies. Such a scheme will be presented in a forthcoming paper.

In this contribution we develop an adequate averaging strategy that revises the existence of the RVE for softening materials. The idea is to average the stresses and strains in the micro samples over a propagating damaged zone rather than over the entire micro sample which is usually the case in standard averaging techniques. A somewhat similar approach in the context of micromechanics can be found in the works of [23, 24]. Having a positive answer on the existence of an RVE for softening materials, a procedure to find its size can be introduced. This could be achieved by a statistical analysis of numerically loaded specimens as presented in [5]. This is however beyond the scope of the paper and interested readers are referred to [25, 26, 27] for details.

As a byproduct of the proposed averaging method, objective homogenized stress-strain relationships which look like traction-separation laws have been obtained. Therefore, the method presented in this work could be utilized either in numerical homogenization based multiscale methods or in computational homogenization based methods for macroscale crack modelling. According to the former, a traction-separation law, which is obtained from microscale finite element (FE) computations via the proposed averaging method in a pre-processing step, is used for describing the behaviour of cracks in a macroscale discrete crack FE model. In the latter, the traction-separation law for a macro crack is determined on the fly, in the spirit of the $\mathrm{FE}^{2}$ method [3], from the responses in a propagating damaged zone at the microscale. Potential application of the proposed averaging scheme can be found in methods with nested scales computations [19, 20, 17] and with domain decomposition methods [28, 29].

The structure of the rest of the paper is as follows. In Section 2 the problem statement is given with details concerning the generation of the samples, the utilized constitutive model and the boundary conditions. The next section presents the proposed averaging scheme followed by a statistical analysis.

\section{Problem statement}

\subsection{Sample generation}

In this contribution, the random heterogeneous material that we investigate is a three-phase material with matrix, aggregates (of circular shape) and an interfacial transition zone (ITZ) surrounding each aggregate. The material samples have been generated using SPACE [30], a discrete element program, with input including the sample size, the size distribution of the aggregates and the density $\rho$ (volume fraction) of aggregates. Details on the generation procedure can be found in [26]. These samples are then discretized into finite element meshes (three noded triangular elements). Figure 1 shows an example where three phases can be distinguished in the close-up view.

In order to study the effect of the size of the samples on the response of the material, for three aggregate densities $\rho(30 \%, 40 \%$ and $60 \%)$, five samples of a size varying from $10 \times 10 \mathrm{~mm}^{2}$ to $30 \times 30 \mathrm{~mm}^{2}$ as depicted in Fig. 2 are 

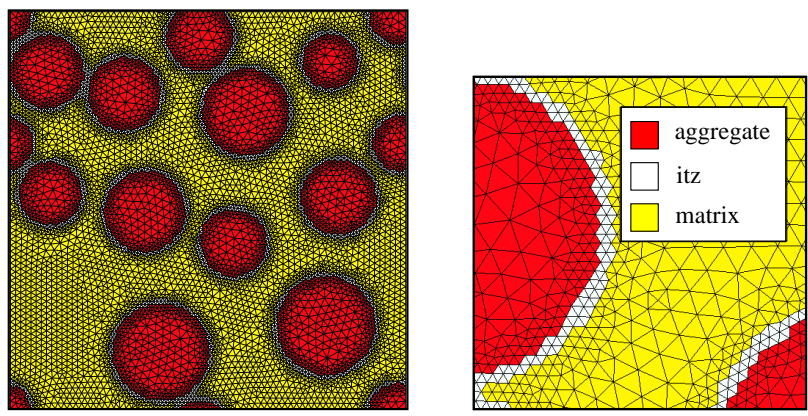

Figure 1: Finite element mesh of the three-phase material sample: full view (left) and close-up view (right).

studied. Sizes of aggregates are varied from $2.5 \mathrm{~mm}$ to $5.0 \mathrm{~mm}$ with a uniformly random distribution. The width of the ITZ is $0.25 \mathrm{~mm}$. The randomness of the aggregate distribution is studied by considering four realizations of each sample size, see Fig. 3 It should be emphasized that four is a small number for a statistical analysis. However it was not the intention of the authors to perform a full statistical analysis, instead a statistical study is given to support the

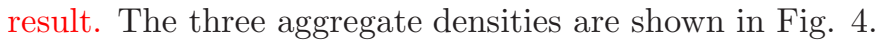

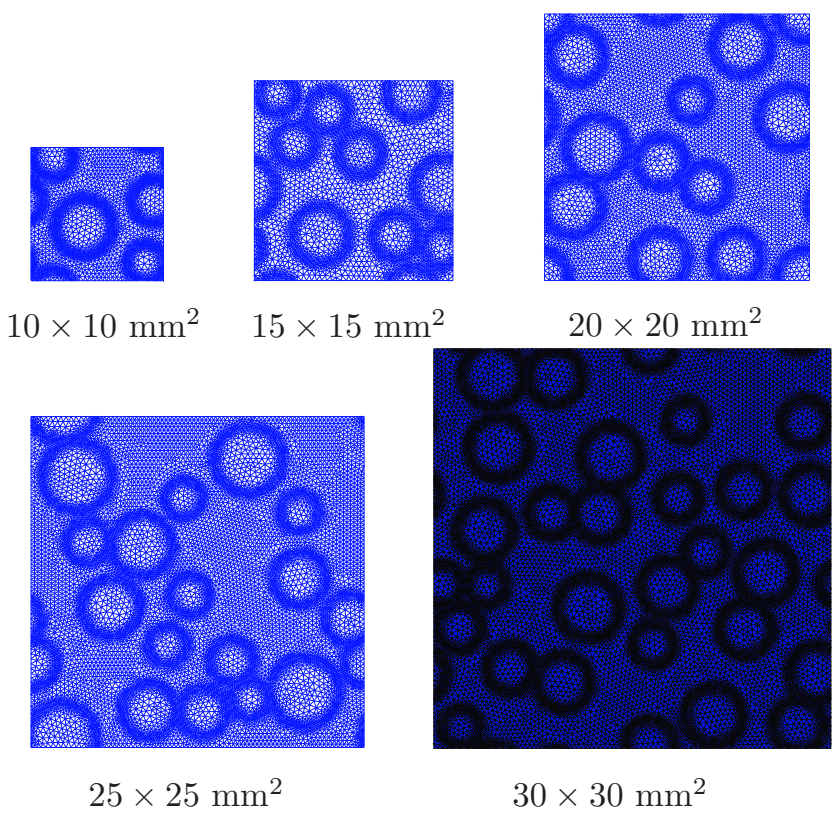

Figure 2: Samples of varying sizes for the case of $30 \%$ aggregate. 

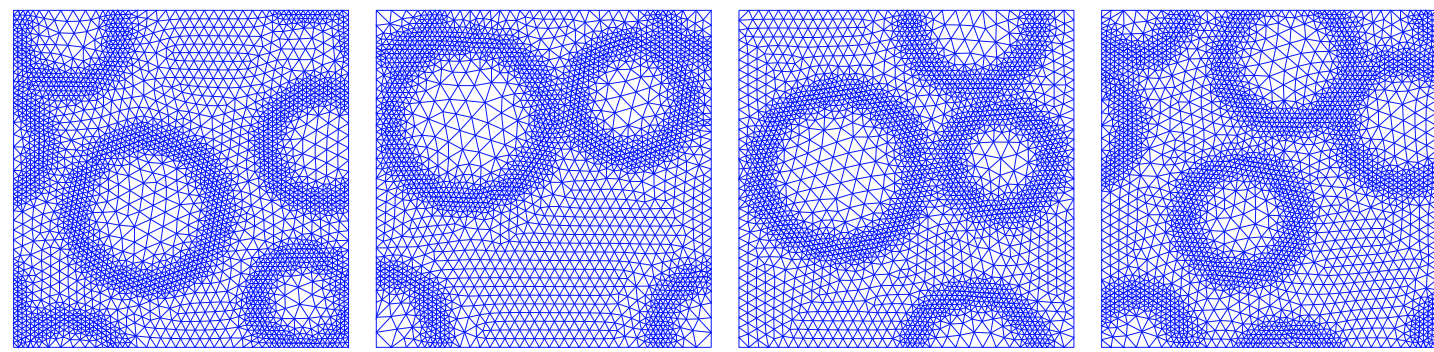

Figure 3: Various realizations of the $10 \times 10 \mathrm{~mm}^{2}$ sample of $30 \%$ aggregate.
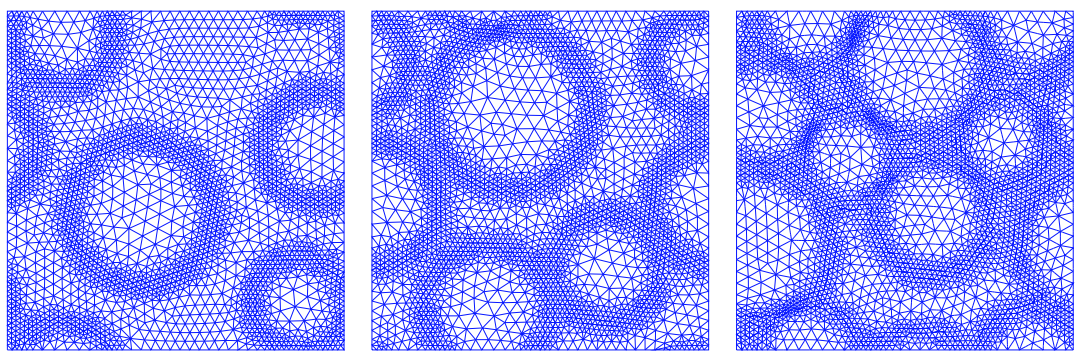

Figure 4: Three aggregate densities studied, from left to right, $\rho=30 \%, \rho=45 \%$ and $\rho=60 \%$ (sample $10 \times 10 \mathrm{~mm}^{2}$ ).

\subsection{Constitutive models}

Damage of the samples is modelled by a simple isotropic damage model regularized by the gradient enhanced method [31]. For sake of completeness, this model is summarized here. The stress-strain relation is given by [32]

$$
\boldsymbol{\sigma}=(1-\omega) \mathbf{D}: \boldsymbol{\epsilon}
$$

where $\omega$ is the scalar damage variable and the second order tensor $\mathbf{D}$ contains the elastic moduli. Damage is governed by the following exponential law

$$
\omega=1-\frac{\kappa}{\kappa_{I}}\left[1-\alpha+\alpha \exp ^{-\beta\left(\kappa-\kappa_{I}\right)}\right], \quad \kappa \geq \kappa_{I}
$$

where $\alpha$ (residual stress), $\beta$ (softening slope) and $\kappa_{I}$ (damage threshold) denote the inelastic parameters. The variable $\kappa$ is a scalar measure of the largest strain ever reached which is defined through the following loading function $f$

$$
f=\bar{\epsilon}_{\mathrm{eq}}-\kappa
$$

with $\bar{\epsilon}_{\text {eq }}$ being the nonlocal equivalent strain which is, according to the gradient enhanced model [31], given by

$$
\bar{\epsilon}_{\mathrm{eq}}-c \nabla^{2} \bar{\epsilon}_{\mathrm{eq}}=\epsilon_{\mathrm{eq}}
$$

where $c$ denotes a positive valued parameter of the dimension length squared and the local equivalent strain $\epsilon_{\mathrm{eq}}$ is defined as [33]

$$
\epsilon_{\mathrm{eq}}=\sqrt{\left\langle\epsilon_{1}\right\rangle^{2}+\left\langle\epsilon_{2}\right\rangle^{2}}
$$


wherein $\epsilon_{i}$ represents the principal strain and the Macauley's brackets $\langle x\rangle$ denote the positive part of $x$.

In summary, the utilized constitutive model is fully characterized by six material parameters: the Young's modulus $E$, the Poisson's ratio $\nu$, the three inelastic parameters $\alpha, \beta, \kappa_{I}$ and the length scale $c$. These material parameters are tabulated in table 1 along with the values taken for the various constituents. Note that $\kappa_{I}$ has been set large for the aggregates to prevent damage and the $c$ parameter has been assumed to be the same for all constituents. The material constants are chosen so that the material consists of hard elastic aggregates embedded in a soft damageable matrix with the ITZ as the weakest region. This could be representative for concrete at mesoscale.

\begin{tabular}{lllll}
\hline & & Matrix & Aggregate & ITZ \\
\hline & & & & \\
$E$ & {$\left[\mathrm{~N} / \mathrm{mm}^{2}\right]$} & 25000 & 30000 & 20000 \\
$\nu$ & {$[-]$} & 0.2 & 0.2 & 0.2 \\
$\kappa_{I}$ & {$[-]$} & $5 \mathrm{e}-06$ & 0.5 & $3 \mathrm{e}-06$ \\
$\alpha$ & {$[-]$} & 0.9 & 0.9 & 0.9 \\
$\beta$ & {$[-]$} & 1500 & 1500 & 1500 \\
$c$ & 0.2 & 0.2 & 0.2 \\
\hline
\end{tabular}

Table 1: Material parameters of different phases.

Remark 2.1. The averaging equation 4 was applied with vanishing normal gradient of the nonlocal equivalent strain as a boundary condition imposed on the external boundary only. Consequently there are non-local interactions between points belonging to different phases. However this issue is not critical in the present paper since the aggregates are elastic and the non-local interactions between the matrix and the ITZ relate to almost similar materials. Interested readers are referred to [34] for related work. We do believe that the conclusions from this paper also apply if the issue of non-locality across material interfaces has been taken into account.

Remark 2.2. The adopted nonlocal damage model introduces an additional material parameter, the so-called internal length scale $\sqrt{c}$. This parameter is supposed to represent phenomena occurring at a lower scale. Considering the heterogeneity nature of the matrix (cement paste) at the microscale (unhydrated cement particles, hydration products and pores), it is reasonable to link $\sqrt{c}$ to the microstructure of the matrix. However this link is yet unknown, the value for $c$ as given in table 1 has been chosen without consideration of the microstructure of the matrix.

The failure process of the material obtained with the continuum damage model used in this work is given in Fig. 5. It starts with damage initiation in the ITZ, then the bulk matrix starts to damage. In later stages, damage zones coalesce to eventually form a localization band.

\subsection{Boundary conditions}

An important aspect in RVE-based homogenization methods is the boundary conditions (BCs), which are imposed on the RVE's boundary, used to capture the effect of the surrounding medium. Commonly used BCs include (i) linear displacements, (ii) uniform traction and (iii) periodic BCs, refer to 35] for a computational treatment of all three BCs. The choice of BCs affects the result of homogenization methods including homogenized properties i.e., macroscale constitutive response, the required size of the RVE and the type (and extent) of localized failure taking place at the microscale. Since linear displacement BCs inhibit localization to occur at the RVE's boundary (because it maps a straight line in an undeformed RVE to a straight line under deformation), it should not be used for softening RVEs. Uniform BCs are also left out of consideration because of the standard strain-driven FE procedure pursued in this work. 

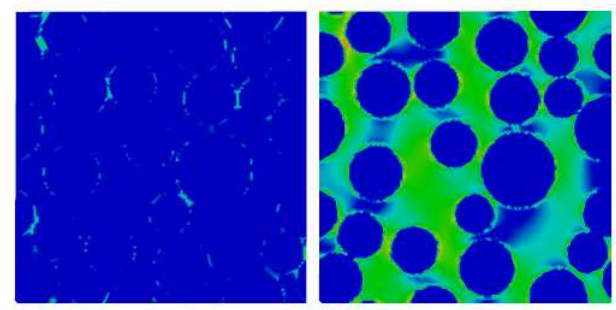

damage
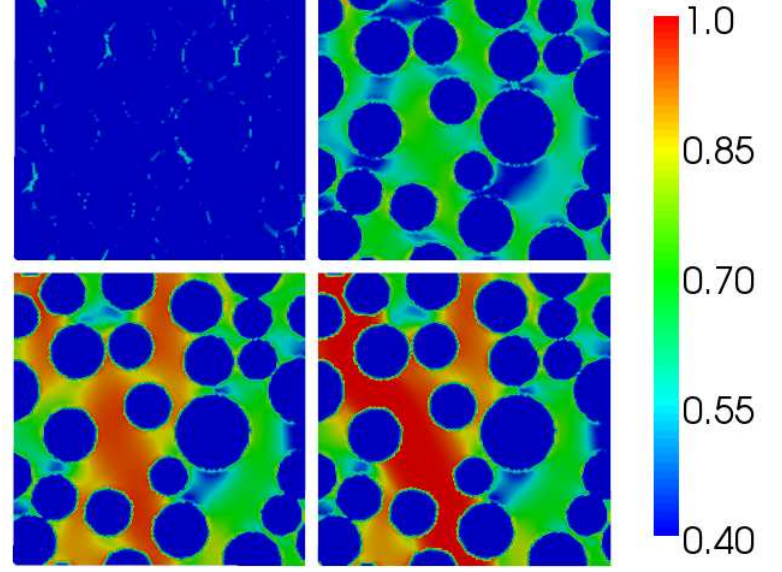

Figure 5: Failure process occurring in one sample under uniaxial tension.
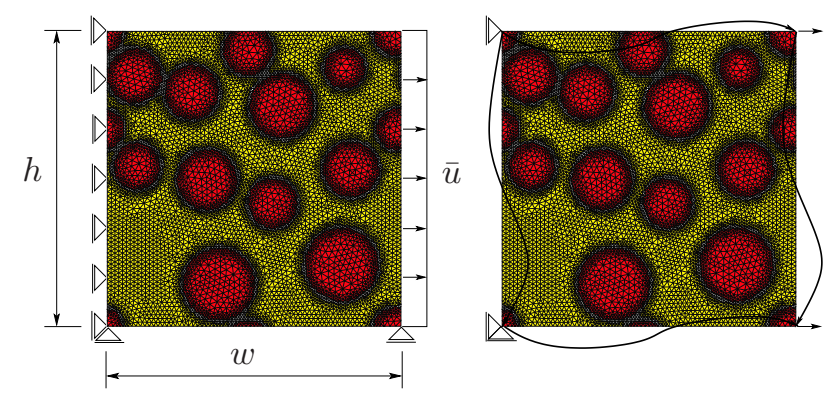

Figure 6: Boundary conditions: utilized (left) and periodic boundary conditions (right). 
In this paper we utilize, unless otherwise stated, the standard BCs as shown in Fig. 6for a uniaxial tension test. Note that this boundary condition has been used in [26] for a study concerning the RVE's size determination. Furthermore, the main content of the current manuscript is not about the proper choice of BCs in RVE-based modelling. Concerning the damage pattern, the difference between the standard BCs and the periodic BCs is that, with periodic BCs, the localization band is forced to be periodic as illustrated by Fig. 7

Non-periodic Periodic boundary condition

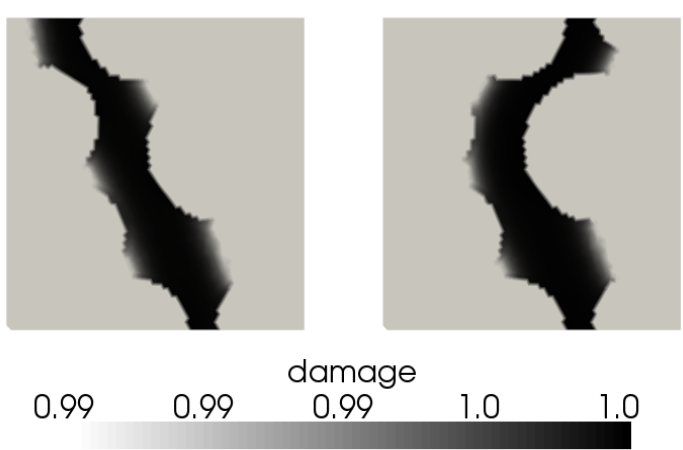

Figure 7: Damage patterns obtained with non-periodic BC and periodic BC.

In all presented computations a plane strain condition has been used. It should be emphasized that under plane strain condition, the circular aggregates represent long cylinders rather than spherical particles. Therefore the material model as adopted in this work was not aiming for as rigorous representative for concrete.

\section{Representative volumes and averaging techniques}

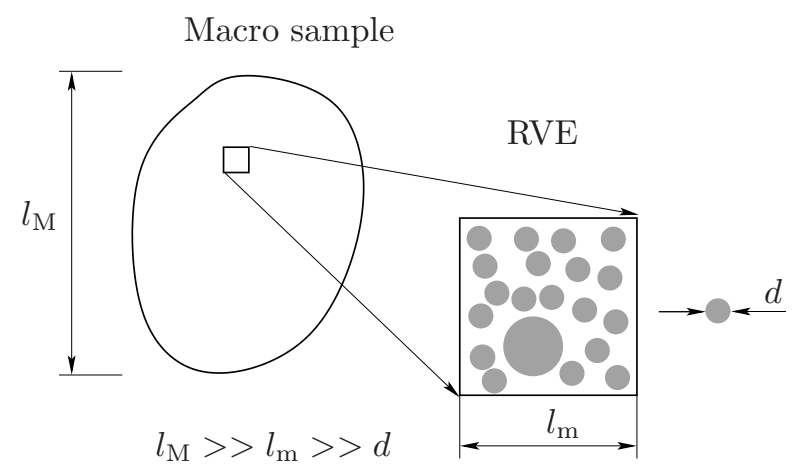

Figure 8: Definition of an RVE for a statistically homogeneous material.

In literature several definitions for the RVE have been proposed for different materials and purposes, refer to [5] for a recent review. For a specific material, the RVE depends on the physical quantity that is measured. Herein we focus our attention on the mechanical response of a material expressed in terms of the averaged stress and strain evolution.

From various existing definitions of the RVE we consider, in the present context of random heterogeneous materials, a microscopic sample to be an RVE when it fulfills all the following requirements 


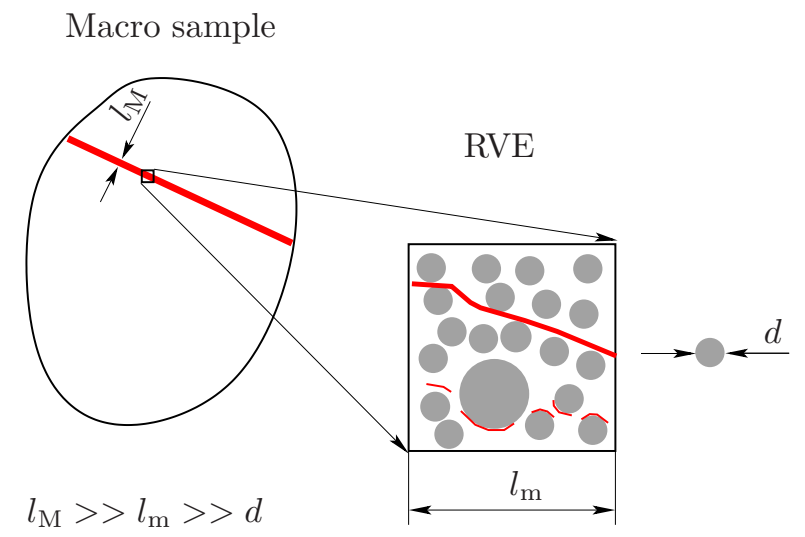

Figure 9: Definition of an RVE for a macroscopic localization zone (thick line). Note that the RVE does not represent (in its classical meaning) the whole material. It does represent for the material on the localization zone.

- an increase in its size does not lead to considerable differences in the homogenized properties

- the microscopic sample is large enough so that the homogenized properties are independent of the microstructural randomness, $l_{\mathrm{m}}>>d$ see Fig. 8

- its size is much smaller than the macroscopic dimension, $l_{\mathrm{M}}>>l_{\mathrm{m}}$ see Fig. 8 This condition is known as the principle of separation of scales.

As has been shown in [5], for quasi-brittle materials, an RVE does exist for the linear and hardening regimes. In the softening regime, however, [5] questioned the existence of the RVE. In this work, we take a closer look at the problem of RVE's existence in the softening regime and we will demonstrate that an RVE does exist in the post-peak regime for softening materials. It should be emphasized that in this case the term RVE means RVE for a localization band (or a crack). Figure 9 gives a graphical illustration for this revised definition of RVE. Note that in this revised definition of the RVE, the macroscopic length scale $l_{\mathrm{M}}$ refers to the size of a macro failure zone.

\subsection{Standard averaging}

The homogenized stresses and strains are defined as the volume averages of its microscopic counterparts, see [36] for details

$$
\begin{aligned}
\langle\boldsymbol{\sigma}\rangle & \equiv \frac{1}{\left|\Omega_{\mathrm{m}}\right|} \int_{\Omega_{\mathrm{m}}} \boldsymbol{\sigma}_{\mathrm{m}} \mathrm{d} \Omega=\frac{1}{\left|\Omega_{\mathrm{m}}\right|} \int_{\Gamma_{\mathrm{m}}} t_{i}^{\mathrm{m}} x_{j}^{\mathrm{m}} \mathrm{d} \Gamma \\
\langle\boldsymbol{\epsilon}\rangle & \equiv \frac{1}{\left|\Omega_{\mathrm{m}}\right|} \int_{\Omega_{\mathrm{m}}} \boldsymbol{\epsilon}_{\mathrm{m}} \mathrm{d} \Omega=\frac{1}{2\left|\Omega_{\mathrm{m}}\right|} \int_{\Gamma_{\mathrm{m}}}\left(u_{i}^{\mathrm{m}} n_{j}^{\mathrm{m}}+u_{j}^{\mathrm{m}} n_{i}^{\mathrm{m}}\right) \mathrm{d} \Gamma
\end{aligned}
$$

where $\Omega_{\mathrm{m}}$ denotes the RVE domain (having volume being $\left|\Omega_{\mathrm{m}}\right|$ ) with the boundary $\Gamma_{\mathrm{m}} ; \boldsymbol{\sigma}_{\mathrm{m}}$ and $\boldsymbol{\epsilon}_{\mathrm{m}}$ are the micro stress and strain tensors, respectively; $\mathbf{t}_{\mathrm{m}}$ represents the micro traction vector while $\mathbf{x}_{\mathrm{m}}$ is the position vector and the unit outward normal to $\Gamma_{\mathrm{m}}$ is given by $\mathbf{n}_{\mathrm{m}}$. 
For the considered boundary conditions, refer to Fig. 6, the homogenized stress and strain along the horizontal direction given in Eq. (6) are simplified to

$$
\begin{aligned}
& \langle\sigma\rangle_{x x}=\frac{f_{\mathrm{R}, \mathrm{x}}^{\mathrm{int}}}{h} \\
& \langle\epsilon\rangle_{x x}=\frac{\bar{u}}{w}
\end{aligned}
$$

where $f_{\mathrm{R}, \mathrm{x}}^{\mathrm{int}}$ is the sum of all nodal internal forces ( $x$ component) along the right edge of the RVE on which an imposed displacement $\bar{u}$ has been applied in the $x$-direction, see Fig. 6 .

A plot of the homogenized normal stress-strain diagrams is given in Fig. 10 for various samples with $30 \%$ aggregates. It is obvious that samples of different sizes behave like different materials e.g., the larger the sample the more brittle it is on average. It has been based on this kind of behavior plus some additional statistical analysis that [5] has questioned the existence of the RVE for softening materials. Although the material model is regularized the response is size dependent. This is caused by the fact that the localization zone does not scale with the size of the RVE, see Fig. 11.

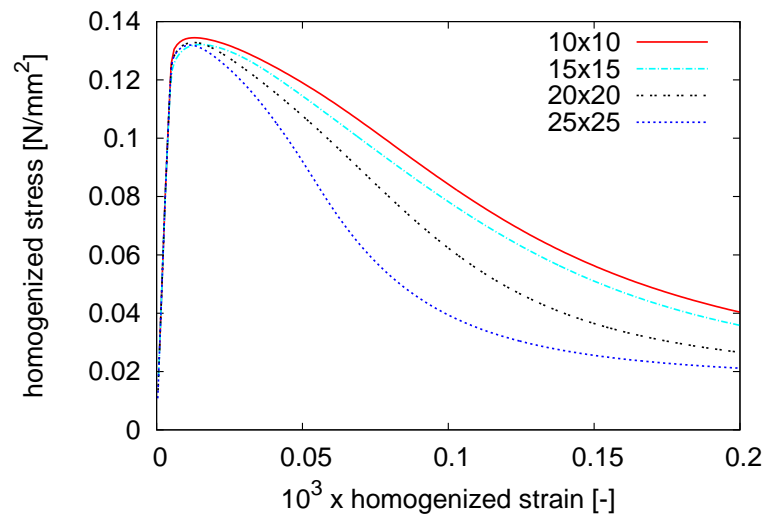

Figure 10: Response of various concrete samples: $30 \%$ aggregate.

\subsection{Failure zone averaging}

The dependency of the homogenized stress-strain diagrams on the RVE size as discussed in the previous section indicates that standard continuum models cannot be adopted at the macroscale in a homogenization-based multiscale framework for softening materials. This observation has called for, over the past few years, the development of the so-called coupled volume methods (see for example [8] and references in the introduction section), the second-order computational homogenization method [37] and coarse graining of failure methods [38, 18, 19, 20, 17]. The salient feature of coarse graining of failure methods is the introduction of discrete cracks at the macroscale model. We are particularly interested in [17] in which the existence of an RVE for materials undergoing discrete cracking has been demonstrated. The existence of an RVE was indicated by the uniqueness (regardless of the utilized micro sample size) of a macro traction-separation law which has been obtained by averaging the responses along propagating micro discrete cracks. 


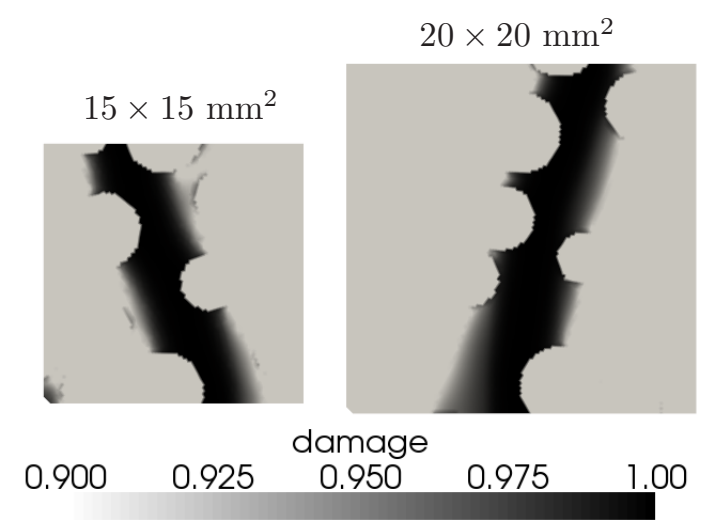

Figure 11: Localization zone does not scale with RVE size (30\% aggregate).

Prompted by the work given in [17] and the fact that the localization band does not scale with the micro sample size (as can be seen in Fig. 11), in this section, we will present an averaging technique by which homogenized stress-strain relationships are obtained which are objective with respect to the micro sample size.

Let us first denote $\Omega_{\mathrm{d}}$ as the active damaged domain i.e., the region containing Gauss points which are damaged and loading. According to the continuum damage model we are utilizing herein this domain corresponds to Gauss points satisfying $\omega>0$ and $f=0$, mathematically $\Omega_{\mathrm{d}}$ is defined as

$$
\Omega_{\mathrm{d}}=\left\{\mathrm{x} \in \Omega_{\mathrm{m}} \mid \omega(\mathbf{x})>0, f(\mathbf{x})=0\right\}
$$

refer to Fig. 12 for a graphical illustration.

We then define the homogenized stresses and strains as the volume averages of the microscopic stresses and strains, respectively, over $\Omega_{\mathrm{d}}$

$$
\begin{gathered}
\langle\boldsymbol{\sigma}\rangle=\frac{1}{\left|\Omega_{\mathrm{d}}\right|} \int_{\Omega_{\mathrm{d}}} \boldsymbol{\sigma}_{\mathrm{m}} \mathrm{d} \Omega_{\mathrm{d}} \\
\langle\boldsymbol{\epsilon}\rangle=\frac{1}{\left|\Omega_{\mathrm{d}}\right|} \int_{\Omega_{\mathrm{d}}} \boldsymbol{\epsilon}_{\mathrm{m}} \mathrm{d} \Omega_{\mathrm{d}}
\end{gathered}
$$

We refer to this averaging scheme as the failure zone averaging scheme. Since the above domain integrals cannot be converted to surface integrals along $\Gamma_{\mathrm{m}}$, they are computed directly using numerical quadrature. This averaging will filter out the linear contribution which makes the standard stress-strain diagrams sample size dependent.

Remark 3.1. Somehow similar approaches in the context of micromechanics can be found in the works of [23, 24] in which the stresses and strains are averaged over a three dimensional homogeneous elastic media containing either randomly oriented or parallel micro cracks.

As an example to test the above averaging scheme, we consider three unit cells of a material with a simple microstructure shown in Fig. 13] The standard stress-strain curve is given in Fig. 14 whereas the failure homogenized stress-strain diagram is shown in Fig. 14b. Obviously one would conclude that there is no unique size of an RVE while looking at Fig. 14. It is, however, clear that an RVE does exist if Fig. 14p has been used as the decision criterion. 


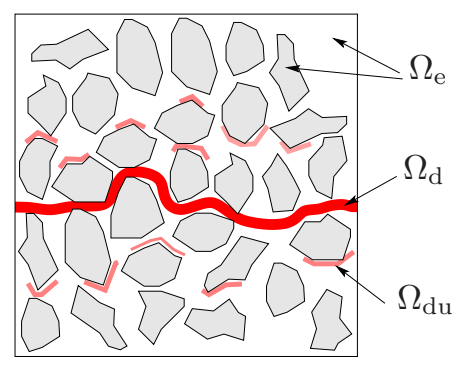

Figure 12: Schematic representation of the active damaged domain $\Omega_{\mathrm{d}}$ over which the averages are performed. Also shown are the elastic domain $\Omega_{\mathrm{e}}$ and the unloading damaged domain $\Omega_{\mathrm{du}}$.

It should be noticed that Eq. (9) should be used only after development of a failure band i.e., after the peak. That explains the absence of a linear branch in the curves shown in Fig. 14b. Figure 15plots the evolution of the damaging area $\left|\Omega_{\mathrm{d}}\right|$ against the failure averaged normal strain. An attempt was also made in which the domain $\Omega_{\mathrm{d}}$ was on purpose incorrectly defined as $\Omega_{\mathrm{m}} \backslash \Omega_{\mathrm{e}}$ (the symbol $\backslash$ is the difference operator from the set theory) which is, of course, larger than the correct active damaged domain $\Omega_{\mathrm{d}}$ as defined in Eq.(8) and Fig. 16] depicts the resulting stress-strain curves which are again sample size dependent, although to a smaller extent than for a standard averaging according to Eq.(66). 5 .

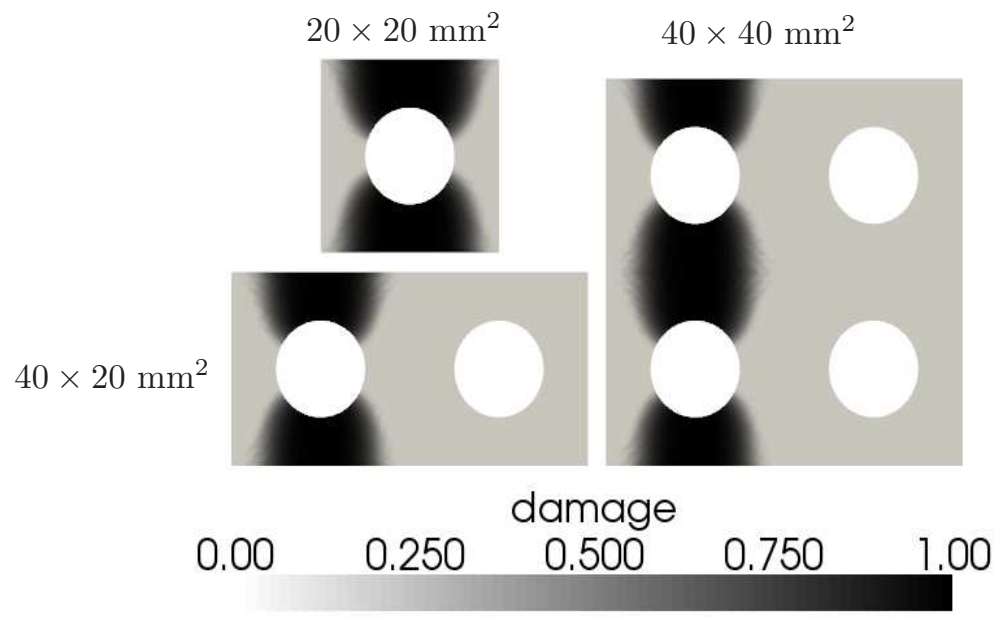

Figure 13: Unit cells of regular microstructure under uniaxial tension in horizontal direction.

\subsection{Representative simulations}

The application of the newly proposed failure zone averaging scheme to the five samples of heterogeneous material, see Fig. 2, is given in Fig. 17 together with the result obtained with the standard averaging method. The curves

\footnotetext{
${ }^{5}$ We have intentionally left the linear part of the curves to emphasize that the failure zone averaging scheme should be used after localization has occurred.
} 


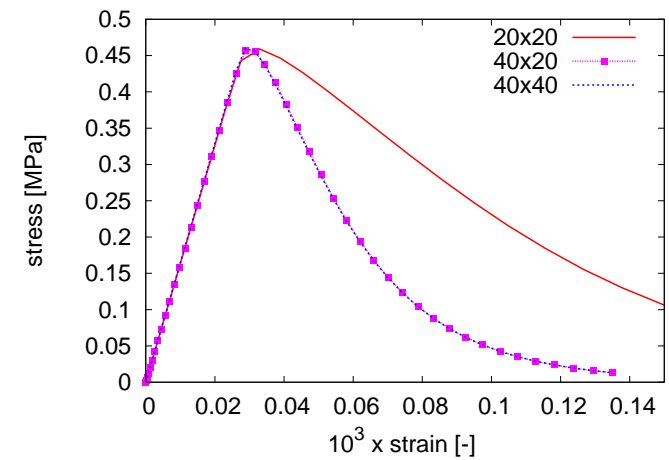

(a) standard averaging

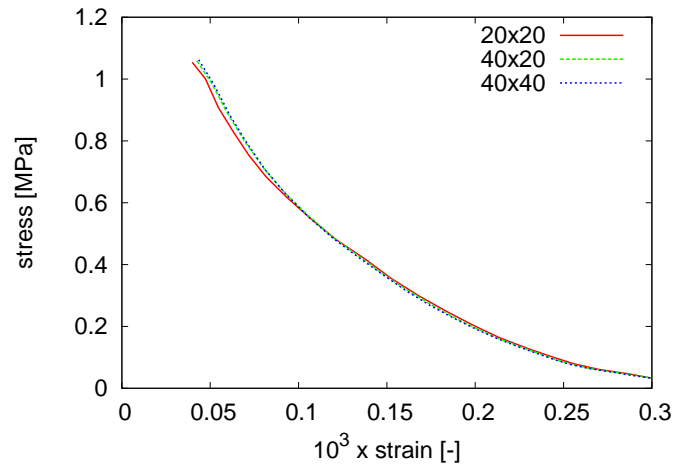

(b) failure averaging

Figure 14: Response of unit cells of regular microstructure.

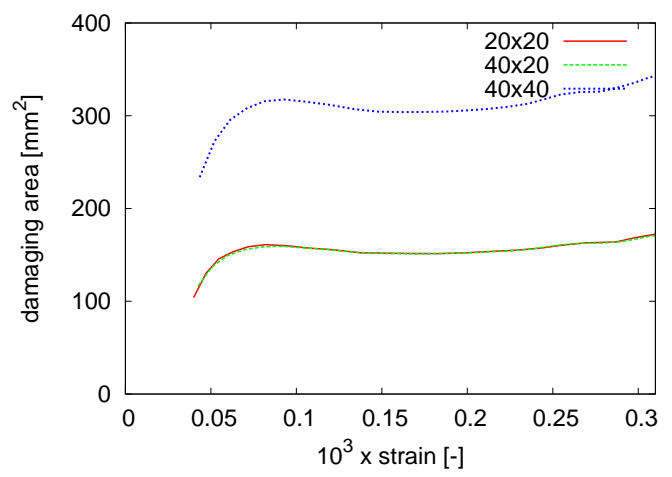

Figure 15: Evolution of the damaging area $\left|\Omega_{\mathrm{d}}\right|$ against the failure averaged normal strain.

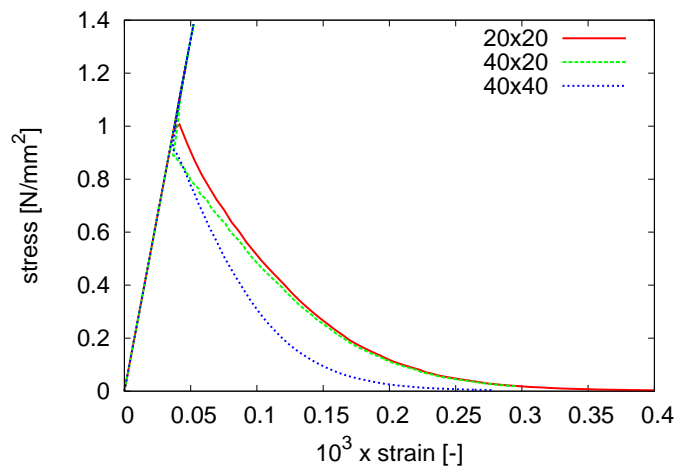

Figure 16: Size-dependent response of three samples made of regular microstructure: the failure zone averaging scheme with $\Omega_{\mathrm{d}}$ incorrectly defined as $\Omega_{\mathrm{m}} \backslash \Omega_{\mathrm{e}}$. 
obviously show that, by filtering out the linear responses in the sample i.e., the stresses and strains in $\Omega_{\mathrm{m}} \backslash \Omega_{\mathrm{d}}$ via the failure zone averaging, the responses are independent of the sample size.

In literature a variety of criteria has been adopted in the statistical analysis of the RVE size namely the slope of the stress-strain curve [7, 5], the dissipated energy [26, 39] and the peak load [26]. In our subsequent statistical analysis we utilize the dissipated energy $G_{f}$ which is the area under the stress-strain curve

$$
G_{f}=\int_{a}^{b}\langle\sigma\rangle_{x x} \mathrm{~d}\langle\epsilon\rangle_{x x}
$$

where the integration limits $a$ and $b$ for the stress-strain curves obtained with the standard averaging scheme are 0 and $0.2 \times 10^{-3}$, respectively see Fig. $17 \mathrm{~h}$. For those computed using the new failure zone averaging scheme, the integration limits $a$ and $b$ are, respectively, the peak value and $1.5 \times 10^{-3}$, see Fig. 17b.

The stress-strain diagrams obtained with the proposed failure averaging scheme of four realizations per sample for the five sample sizes (see Fig. 2) corresponding to $\rho=45 \%$ are given in Fig. 18. Those obtained with the standard averaging are not reproduced here since they were given in [7, 5]. These curves are the input for the subsequent statistical analysis with the expectation and the standard deviation of a variable $x$ (herein $G_{f}$ ) given by

$$
\bar{x}=\frac{1}{n} \sum_{i=1}^{n} x_{i}, \quad s=\frac{1}{n-1} \sum_{i=1}^{n}\left(x_{i}-\bar{x}\right)^{2}
$$

with $n$ being the number of realizations for each sample and $x_{i}$ is the variable associated with the sample $i$.

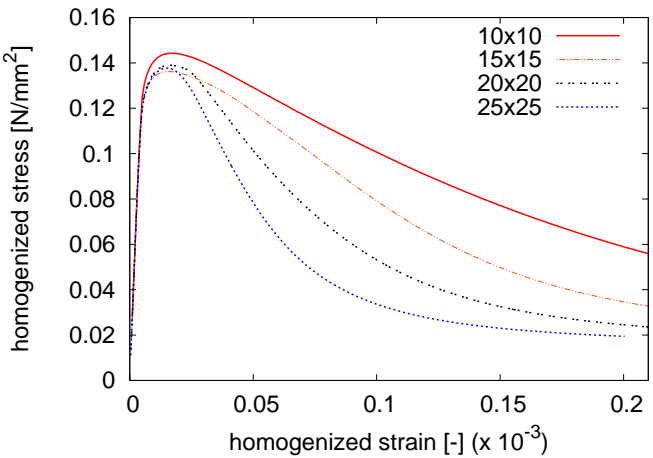

(a) standard averaging

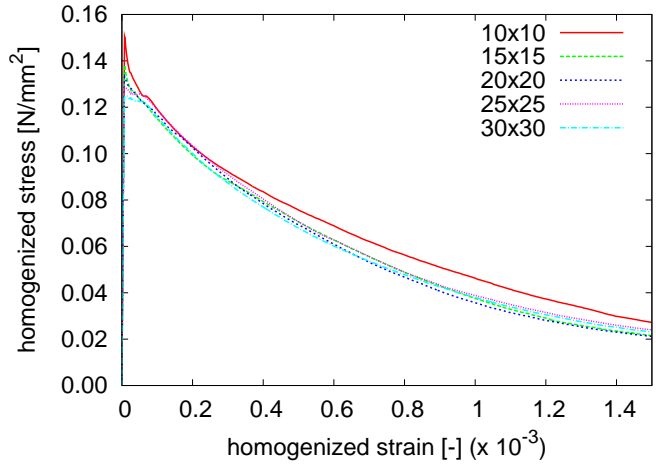

(b) failure averaging

Figure 17: Response of various samples made of random microstructure $(\rho=45 \%)$.

The statistical analysis result is given in Fig. 19 in which the left figure reproduced the result reported in [7, 5] for the sake of comparison. In Fig. 19a we can see that the deviation converges upon increasing sample size, the expectation however steadily decreases which again demonstrates the RVE size dependence. Figure 19b shows, however, that the expectation stabilizes while the deviation shows a decreasing tendency upon increasing sample size which supports the RVE size independence of the failure zone averaging method. In Fig. 20, the results for $\rho=30 \%$ and $\rho=60 \%$ are given which shows the same trend. 


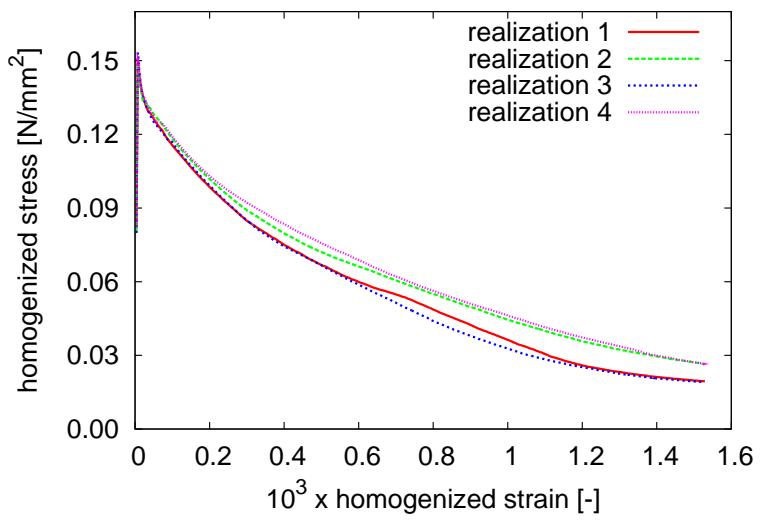

(a) $10 \times 10$

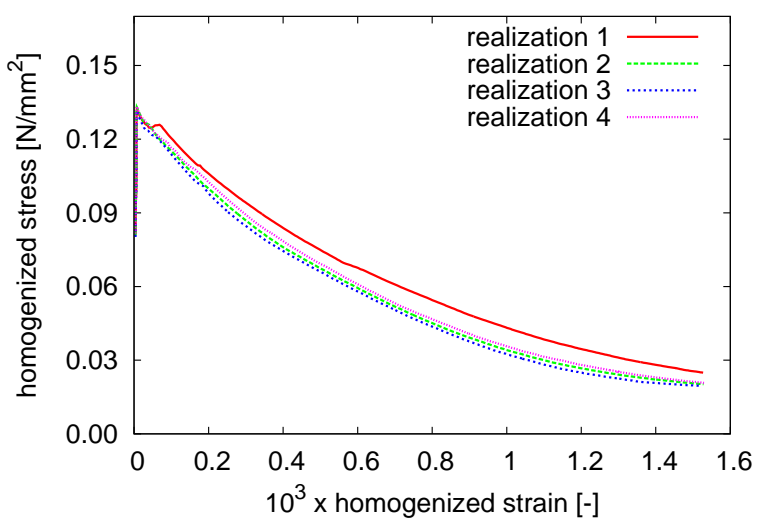

(c) $20 \times 20$

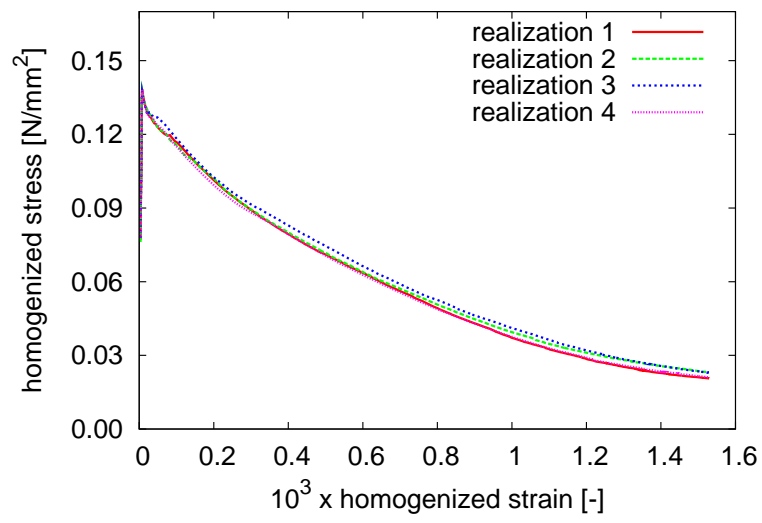

(b) $15 \times 15$

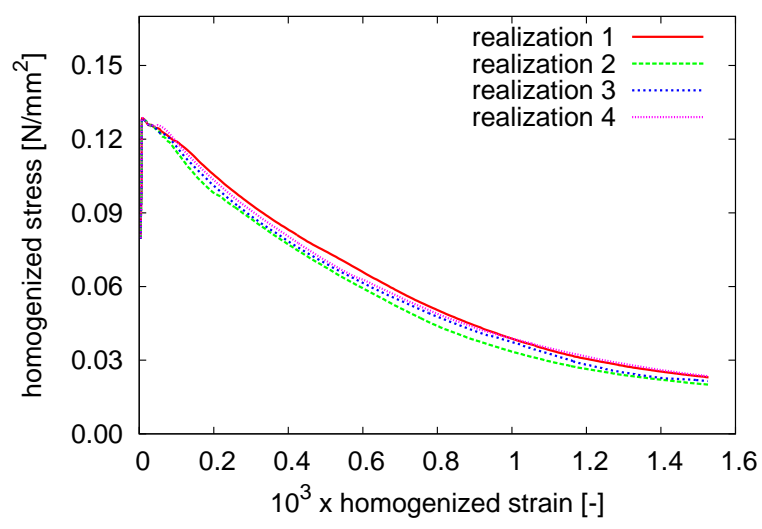

(d) $25 \times 25$

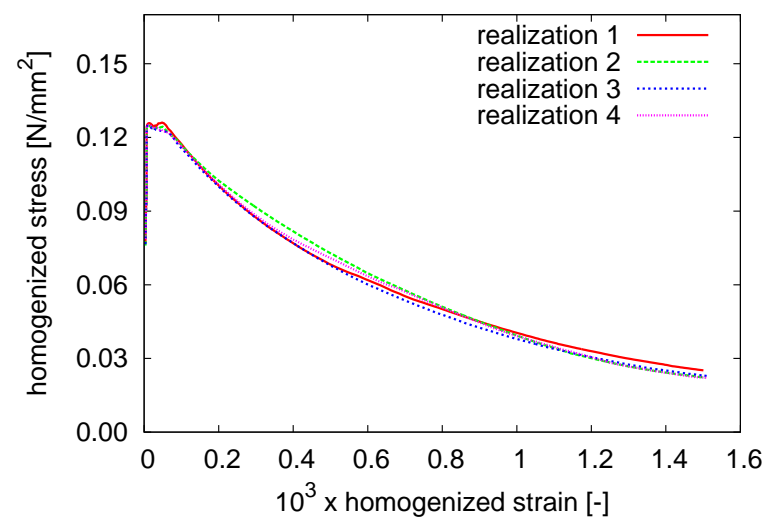

(e) $30 \times 30$

Figure 18: Failure averaged stress-strain curves of realizations of various samples $(\rho=45 \%)$. 


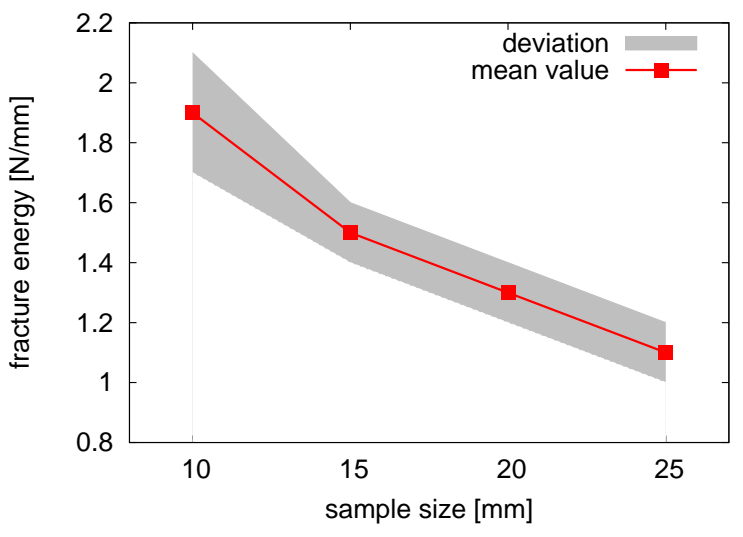

(a) standard averaging

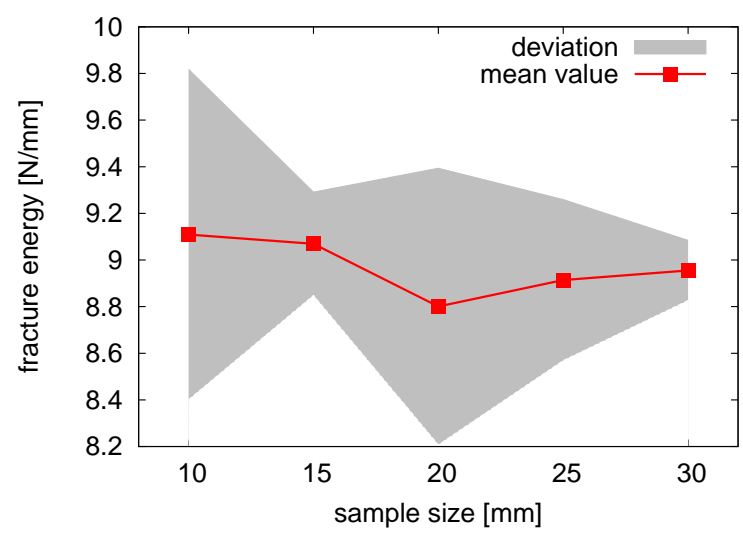

(b) failure averaging

Figure 19: Mean value and standard deviation of the dissipated energy (scaled by $10^{5}$ ) for $\rho=45 \%$.

\subsection{A note on periodic boundary conditions}

In this section, instead of using the standard BCs given in the left figure of Fig. 6, periodic BCs (right figure of Fig. (6) are used. It should be emphasized that the aim is not to perform a comparative analysis on those two BCs. Instead we would like to stress the objectivity of the failure zone averaging technique with respect to the BCs. To this end, only $\rho=45 \%$ is considered and five samples (one realization per sample) are analysized. The results given in Fig. 21 indicate that with the failure zone averaging scheme responses which are independent of the micro sample size are obtained also for periodic BCs.

\subsection{Preliminary result for shear loading}

This section discusses the analyses for five samples (one realization per sample) in case of $\rho=45 \%$ subjected to a shear loading as shown in Fig. 22 The result shown in Fig. 23 confirms the independence of the homogenized responses obtained with the proposed averaging scheme with respect to the micro sample size. Although this is a promising result, we believe that a statistical analysis as performed for the uniaxial tension test is needed before a claim on the existence of an RVE for softening materials in shear can be stated.

\section{Conclusions}

In this paper the issue of existence of the representative volume element for softening quasi-brittle materials with random microstructure has been studied. By performing the averaging over the active damaged domain, rather than the entire domain, by which the linear contribution has been filtered out, convergence of the stress-strain diagrams for different RVE sizes of softening granular materials has been obtained for tensile loading. Extension of this work will be $(i)$ the verification of the existence of the RVE for shear loading and mixed-mode loading, (ii) the statistically proper determination of the RVE's size for softening materials. The existence of an RVE for softening materials represents a necessary starting point for the hierarchical multiscale scheme for softening materials based on computational homogenization techniques. 


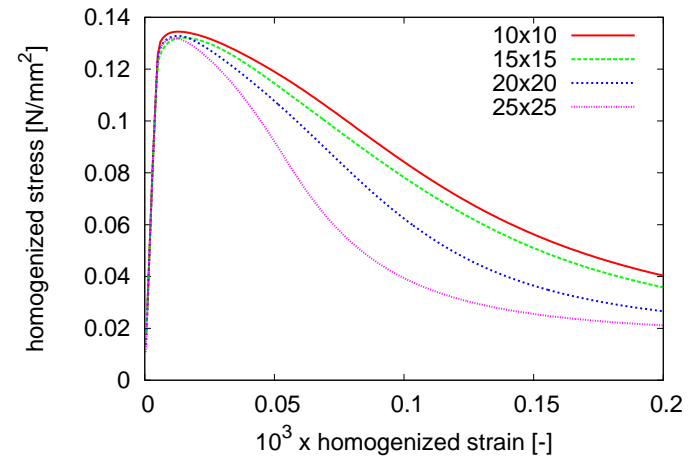

(a) standard averaging

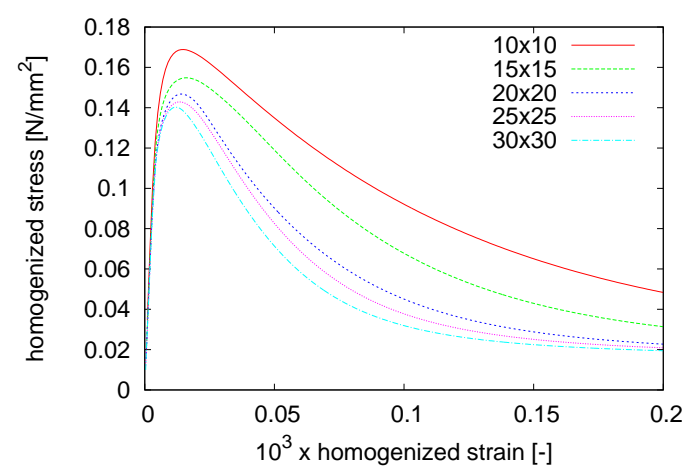

(c) standard averaging

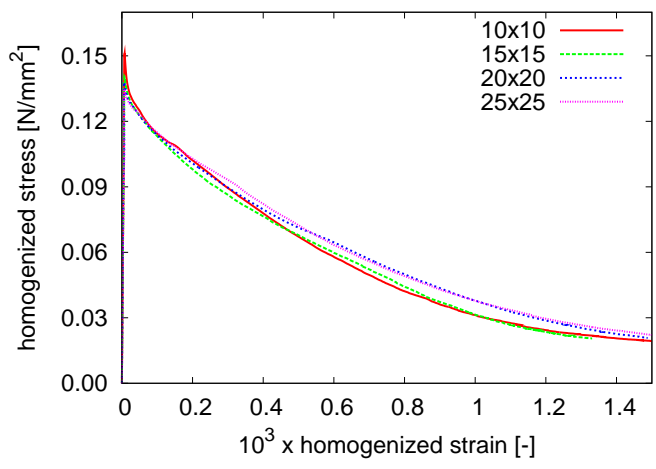

(b) failure averaging

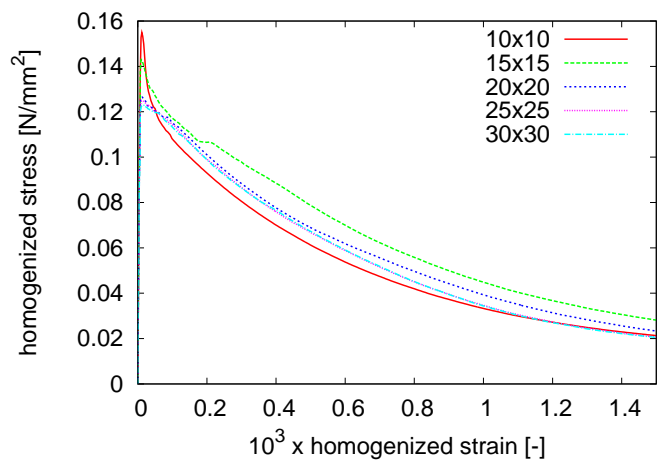

(d) failure averaging

Figure 20: Response of various samples made of random microstructure for $\rho=30 \%$ (top) and for $\rho=60 \%$ (bottom). 


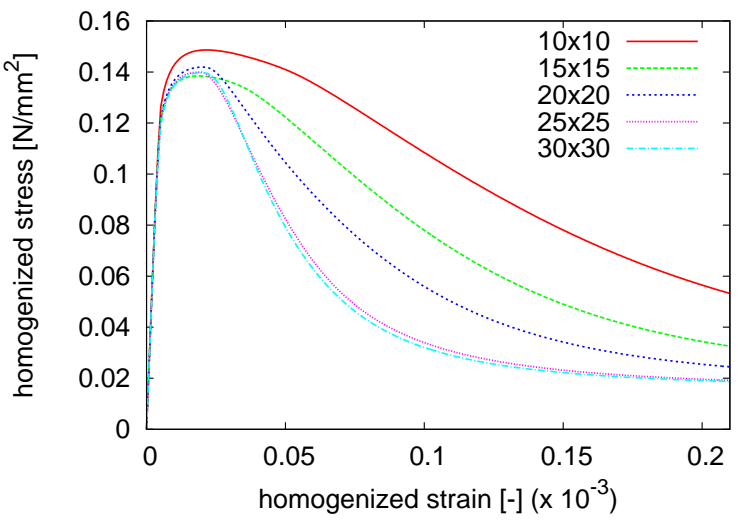

(a) standard averaging

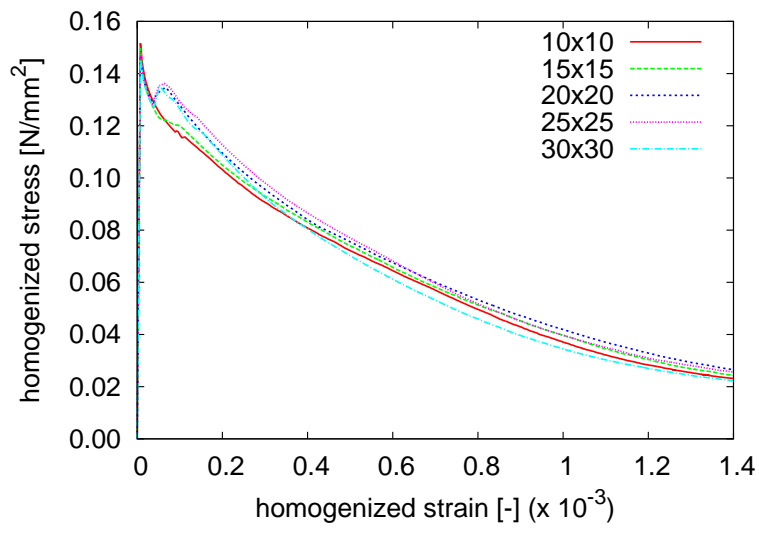

(b) failure averaging

Figure 21: Response of samples made of random microstructure $(\rho=45 \%)$ with periodic boundary conditions.

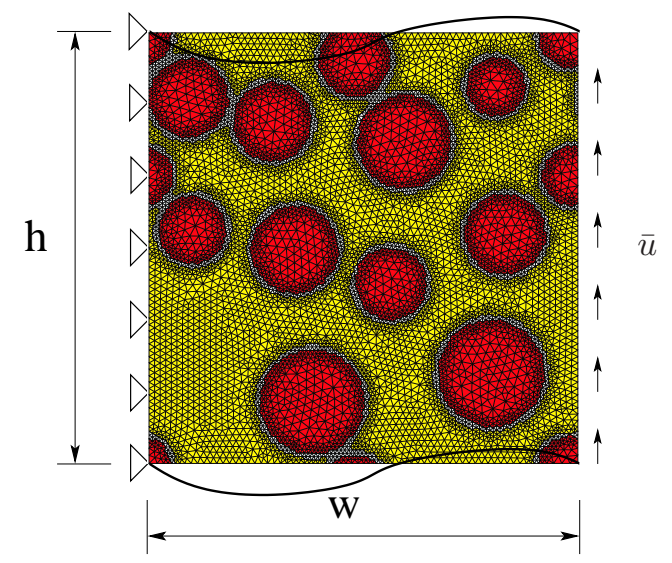

Figure 22: Micro sample subjected to a shear loading. 


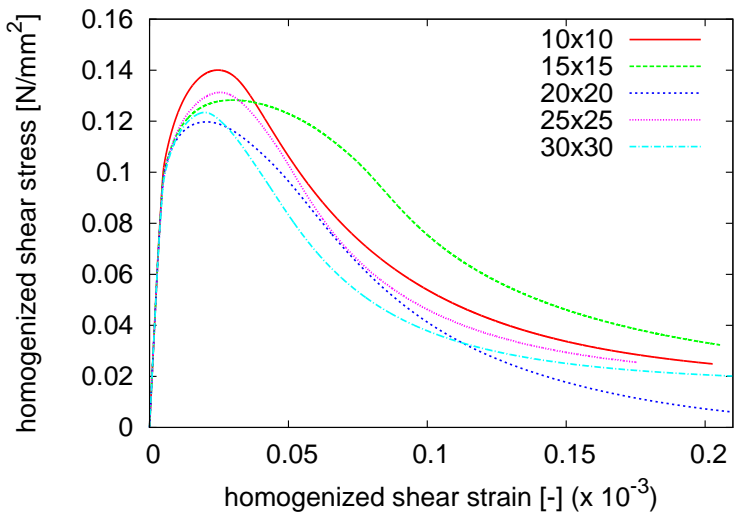

(a) standard averaging

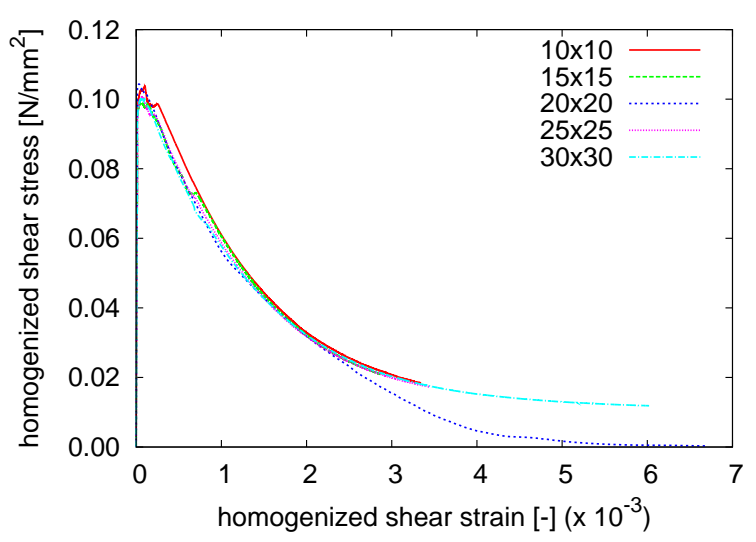

(b) failure averaging

Figure 23: Responses of samples made of random microstructure $(\rho=45 \%)$ in shear loading.

\section{Acknowledgements}

The financial support from the Delft Center for Computational Science and Engineering (DCSE) is gratefully acknowledged.

\section{References}

[1] R.J.M. Smit, W.A.M. Brekelmans, and H.E.H. Meijer. Prediction of the mechanical behavior of nonlinear heterogeneous systems by multi-level finite element modeling. Computer Methods in Applied Mechanics and Engineering, $55: 181-192,1998$.

[2] S. Ghosh, K. Lee, and S. Moorthy. Two scale analysis of heterogeneous elastic-plastic materials with asymptotic homogenization and voronoi cell finite element model. Computer Methods in Applied Mechanics and Engineering, 132:63-116, 1996.

[3] F. Feyel. A multilevel finite element method (fe2) to describe the response of highly non-linear structures using generalized continua. Computer Methods in Applied Mechanics and Engineering, 192:3233-3244, 2003.

[4] V. Kouznetsova, W. A. M. Brekelmans, and F. P. T. Baaijens. An approach to micro-macro modeling of heterogeneous materials. Computational Mechanics, 27(1):37-48, 2001.

[5] I.M. Gitman, H. Askes, and L.J. Sluys. Representative volume: Existence and size determination. Engineering Fracture Mechanics, 74(16):2518-2534, 2007.

[6] S. Graham and N. Yang. Representative volumes of materials based on microstructural statistics. Scripta Materialia, 48(3):269-274, 2003.

[7] I.M. Gitman, H. Askes, L.J. Sluys, and O. Lloberas. The concept of representative volume for elastic, hardening and softening materials. In Proceedings of XXXII International Summer School-Conference "Advance problems in Mechanics", pages 180-184, Saint Petersburg (Repino) Russia, 2004. 
[8] I. M. Gitman, H. Askes, and L. J. Sluys. Coupled-volume multi-scale modelling of quasi-brittle material. European Journal of Mechanics - A/Solids, 27(3):302-327, 2007.

[9] A. Ibrahimbegovic and D. Markovic. Strong coupling methods in multi-phase and multi-scale modeling of inelastic behavior of heterogeneous structures. Computer Methods in Applied Mechanics and Engineering, 192(28-30):3089$3107,2003$.

[10] A. Hund and E. Ramm. Locality constraints within multiscale model for non-linear material behaviour. International Journal for Numerical Methods in Engineering, 70(13):1613-1632, 2007.

[11] T. Hettich, A. Hund, and E. Ramm. Modeling of failure in composites by X-FEM and level sets within a multiscale framework. Computer Methods in Applied Mechanics and Engineering, 197(5):414-424, 2008.

[12] S. Eckardt and C. Könke. Adaptive damage simulation of concrete using heterogeneous multiscale models. Journal of Algorithms \&3 Computational Technology, 2:275-297, June 2008.

[13] O. Lloberas Valls, D. J. Rixen, A. Simone, and L. J.Sluys. A domain decomposition approach to multiscale analysis for structures with softening materials. In J. Ambrosio et.al., editor, Proceedings of the 7th EUROMECH Solid Mechanics Conference - ESMC2009, Lisbon, Portugal, 7 - 11 September 2009.

[14] K. Matouš, M. G. Kulkarni, and P. H. Geubelle. Multiscale cohesive failure modeling of heterogeneous adhesives. Journal of the Mechanics and Physics of Solids, 56(4):1511-1533, 2008.

[15] C. B. Hirschberger, S. Ricker, P. Steinmann, and N. Sukumar. Computational multiscale modelling of heterogeneous material layers. Engineering Fracture Mechanics, 76(6):793-812, 2009.

[16] M.V. Cid Alfaro, A.S.J. Suiker, C.V. Verhoosel, and R. de Borst. Numerical homogenization of cracking processes in thin fibre-epoxy layers. European Journal of Mechanics - A/Solids, In Press, Corrected Proof, 2010.

[17] C. V. Verhoosel, J. J. C. Remmers, M. A. Gutiérrez, and R. de Borst. Computational homogenisation for adhesive and cohesive failure in quasi-brittle solids. International Journal for Numerical Methods in Engineering, 2010.

[18] T. Belytschko, S. Loehnert, and J.H. Song. Multiscale aggregating discontinuities: A method for circumventing loss of material stability. International Journal for Numerical Methods in Engineering, 73(6):869-894, 2008.

[19] T.J. Massart and B.C.N. Mercatoris. Assessment of periodic homogenisation-based multiscale computational schemes for quasi-brittle structural failure. International Journal for Multiscale Computational Engineering, $7(2): 153-170,2009$.

[20] T. Belytschko and J.H. Song. Coarse-graining of multiscale crack propagation. International Journal for Numerical Methods in Engineering, 81(5):537-563, 2010.

[21] Julien Sanahuja and Luc Dormieux. Résistance d'un milieu poreux à phase solide hétérogène. Comptes Rendus Mécanique, 333:818-823, November 2005.

[22] Andreas Fritsch, Luc Dormieux, Christian Hellmich, and Julien Sanahuja. Micromechanics of crystal interfaces in polycrystalline solid phases of porous media: fundamentals and application to strength of hydroxyapatite biomaterials. Journal of Materials Science, 42(21):8824-8837, November 2007.

[23] L. Dormieux, D. Kondo, and F.J. Ulm. A micromechanical analysis of damage propagation in fluid-saturated cracked media. Comptes Rendus Méchanique, 334(7):440-446, 2006. 
[24] B. Pichler, C. Hellmich, and H. A. Mang. A combined fracture-micromechanics model for tensile strain-softening in brittle materials, based on propagation of interacting microcracks. International Journal for Numerical and Analytical Methods in Geomechanics, 31(2):111-132, 2007.

[25] W. J. Drugan and J. R. Willis. A micromechanics-based nonlocal constitutive equation and estimates of representative volume element size for elastic composites. Journal of the Mechanics and Physics of Solids, 44(4):497-524, 1996.

[26] M. Stroeven, H. Askes, and L. J. Sluys. Numerical determination of representative volumes for granular materials. Computer Methods in Applied Mechanics and Engineering, 193(30-32):3221-3238, 2004.

[27] C. Pelissou, J. Baccou, Y. Monerie, and F. Perales. Determination of the size of the representative volume element for random quasi-brittle composites. International Journal of Solids and Structures, 46(14-15):2842-2855, 2009.

[28] P. Kerfriden, O. Allix, and P. Gosselet. A three-scale domain decomposition method for the 3D analysis of debonding in laminates. Computational Mechanics, 44(3):343-362, 2009.

[29] P. Gosselet and C. Rey. Non-overlapping domain decomposition methods in structural mechanics. Archives of computational methods in engineering, 13(4):515-572, 2006.

[30] M. Stroeven and P. Stroeven. SPACE system for simulation of aggregated matter application to cement hydration. Cement and Concrete Research, 29(8):1299-1304, 1999.

[31] R.H.J. Peerlings, R. de Borst, W.A.M. Brekelmans, and J.H.P. de Vree. Gradient enhanced damage for quasi-brittle materials. International Journal for Numerical Methods in Engineering, 39:3391-3403, 1996.

[32] J. Lemaitre. A course on damage mechanics. Springer-Verlag, 1996.

[33] J. Mazars and G.Pijaudier-Cabot. Continuum damage theory - application to concrete. Journal of Engineering Mechanics Division ASCE, 115:345-365, 1989.

[34] A. Krayani, G. Pijaudier-Cabot, and F. Dufour. Boundary effect on weight function in nonlocal damage model. Engineering Fracture Mechanics, 76(14):2217-2231, September 2009.

[35] C. Miehe and A. Koch. Computational micro-to-macro transitions of discretized microstructures undergoing small strains. Archive of Applied Mechanics, 72(4):300-317, July 2002.

[36] T.I. Zohdi and P. Wriggers. Introduction to computational micromechanics. Springer-Verlag, 2005.

[37] V. Kouznetsova, M. G. D. Geers, and W. A. M. Brekelmans. Multi-scale constitutive modelling of heterogeneous materials with a gradient-enhanced computational homogenization scheme. International Journal for Numerical Methods in Engineering, 54(8):1235-1260, 2002.

[38] T. J. Massart, R. H. J. Peerlings, and M. G. D. Geers. An enhanced multi-scale approach for masonry wall computations with localization of damage. International Journal for Numerical Methods in Engineering, 69(5):1022-1059, 2007.

[39] M. G. Kulkarni, P. H. Geubelle, and K. Matouš. Multi-scale modeling of heterogeneous adhesives: Effect of particle decohesion. Mechanics of Materials, 41(5):573-583, 2009. 